\title{
Phase II study evaluating consolidation whole abdominal intensity-modulated radiotherapy (IMRT) in patients with advanced ovarian cancer stage FIGO III - The OVAR-IMRT-02 Study
}

Nathalie Rochet ${ }^{1 *}$, Meinhard Kieser ${ }^{2}$, Florian Sterzing ${ }^{1}$, Sonja Krause ${ }^{1}$, Katja Lindel ${ }^{1}$, Wolfgang Harms ${ }^{1}$, Michael H Eichbaum ${ }^{3}$, Andreas Schneeweiss ${ }^{4}$, Christof Sohn ${ }^{3}$, Juergen Debus ${ }^{1}$

\begin{abstract}
Background: The prognosis for patients with advanced FIGO stage III epithelial ovarian cancer remains poor despite the aggressive standard treatment, consisting of maximal cytoreductive surgery and platinum-based chemotherapy. The median time to recurrence is less than 2 years, with a 5 -years survival rate of $-20-25 \%$. Recurrences of the disease occur mostly intraperitoneally. Ovarian cancer is a radiosensitive tumor, so that the use of whole abdominal radiotherapy (WAR) as a consolidation therapy would appear to be a logical strategy. WAR used to be the standard treatment after surgery before the chemotherapy era; however, it has been almost totally excluded from the treatment of ovarian cancer during the past decade because of its high toxicity. Modern intensity-modulated radiation therapy (IMRT) has the potential of sparing organs at risk like kidneys, liver, and bone marrow while still adequately covering the peritoneal cavity with a homogenous dose.

Our previous phase I study showed for the first time the clinical feasibility of intensity-modulated WAR and pointed out promising results concerning treatment tolerance. The current phase-II study succeeds to the phase-I study to further evaluate the toxicity of this new treatment.
\end{abstract}

Methods/design: The OVAR-IMRT-02 study is a single-center one arm phase-Il trial. Thirty seven patients with optimally debulked ovarian cancer stage FIGO III having a complete remission after chemotherapy will be treated with intensity-modulated WAR as a consolidation therapy.

A total dose of $30 \mathrm{~Gy}$ in 20 fractions of $1.5 \mathrm{~Gy}$ will be applied to the entire peritoneal cavity including the liver surface and the pelvic and para-aortic node regions. Organ at risk are kidneys, liver (except the $1 \mathrm{~cm}$-outer border), heart, vertebral bodies and pelvic bones.

Primary endpoint is tolerability; secondary objectives are toxicity, quality of life, progression-free and overall survival. Discussion: Intensity-modulated WAR provides a new promising option in the consolidation treatment of ovarian carcinoma in patients with a complete pathologic remission after adjuvant chemotherapy. Further consequent studies will be needed to enable firm conclusions regarding the value of consolidation radiotherapy within the multimodal treatment of advanced ovarian cancer.

Trial registration: Clinicaltrials.gov: NCT01180504

\footnotetext{
* Correspondence: nathalie.rochet@med.uni-heidelberg.de

'Department of Radiation Oncology, University of Heidelberg, Im

Neuenheimer Feld 400, 69120 Heidelberg, Germany

Full list of author information is available at the end of the article
} 


\section{Background}

Ovarian cancer has the highest mortality rate of all gynaecologic cancer in the western world. Symptoms tend to be vague; hence the disease is often detected late. $75 \%$ of patients present with advanced stage disease, after it has spread into the peritoneal cavity (stage FIGO III). Despite the aggressiveness of the current standard treatment, consisting in maximal cytoreductive surgery and adjuvant combination chemotherapy (carboplatin and taxane), the prognosis for patients in a stage FIGO III disease remains poor. The median time to recurrence is less than 2 years. Typically recurrences occur intraperitoneally, distant metastasis appear mostly later in the course of the disease. The 5-year survival rate for stage FIGO III disease is approximately 20-25\% [1-5]. Various sorts of consolidation therapy have been studied in a series of clinical trials, but yet none has shown to improve survival in ovarian cancer [6-8].

The use of whole abdominal radiotherapy (WAR) as a consolidation therapy would appear to be a logical strategy given that ovarian cancer is a radiosensitive tumor. The fact that WAR can be curative in certain groups of patients has been shown by intensive studies by Dembo et al $[9,10]$. However, the role of radiation therapy in ovarian cancer remains controversial. In the 1950s, surgery and adjuvant WAR were the dominant treatment modalities $[9,10]$. Since the improvements of chemotherapy, which gives primarily high response rates and has a systemic effect, radiotherapy has been increasingly abandoned [11].

WAR as a consolidation concept after surgery and chemotherapy has been evaluated in a number of small and non-randomized studies with contradictory results [12-17]. The recent randomized trial of Sorbe indicated that WAR may have a place in the treatment of advanced ovarian carcinoma in patients with complete pathologic remission after adjuvant chemotherapy [18]. A potential benefit was noted regarding the rate of recurrence and progression-free as well as overall survival rates. These results are supported by other small recent series [15,19-23]. It seems that 30 Gy delivered to the whole abdomen can eliminate residual microscopic disease in $45-50 \%$ of the cases [15].

However, there are many reasons why consolidation WAR fell into disfavour. It is associated with a high rate of severe acute side effects, particularly myelosuppression, due to additive toxicities after chemotherapy, which often leads to delayed or incomplete radiotherapy [16]. WAR is ineffective for patients with macroscopic disease residuum after chemotherapy [24]. Using the conventional technique, WAR is not able to deliver adequate radiation doses to the upper abdomen, due to limiting organs at risk (OAR), especially liver and kidneys [9]. Eventually WAR is unpopular because of the late bowel toxicity, which is surgically difficult to manage.
As a consequence, during the past decade, WAR has been almost totally excluded from the treatment of advanced ovarian. However, toxicity can now be managed by modern radiotherapy techniques using intensity-modulated radiotherapy (IMRT), which allow a very precise irradiation of complex target volumes while sparing organs at risk like kidneys, liver, and bone marrow [25-29].

Our previous phase I study showed, for the first time, the clinical feasibility of intensity-modulated WAR in combination with modern chemotherapy and surgery and pointed out promising results concerning treatment tolerance $[26,30,31]$. Ten patients with optimally debulked ovarian cancer FIGO stage IIIc were treated with intensity-modulated WAR up to a total dose of 30 Gy in 1.5 Gy fractions as a consolidation therapy after adjuvant carboplatin/taxane chemotherapy. Treatment was delivered using IMRT in step-and-shoot technique $(n=3)$ or helical tomotherapy technique $(n=7)$. The planning target volume (PTV) included the entire peritoneal cavity and the pelvic and para-aortic node regions. Organs at risk (OARs) were kidneys, liver, heart, vertebral bodies and pelvic bones.

Intensity-modulated WAR rendered an excellent coverage of the entire peritoneal cavity including frequent sites of abdominal recurrence like the liver capsule and the diaphragm as well as of the pelvic- and para-aortic node regions. An effective sparing of liver, kidneys and bone marrow allowed delivering the treatment as planned, with a high compliance of patients. Radiotherapy was well tolerated, no severe acute side effects CTC grade 4 occurred. CTC grade 3 toxicities were: diarrhea $(n=1)$, thrombocytopenia $(n=1)$ and leukopenia $(n=3)$. After a median follow-up of 23 months, 4 patients had a tumor recurrence (intraperitoneal progression $\mathrm{n}=3$, hepatic metastasis $\mathrm{n}=1$ ). Small bowel obstruction due to adhesions occurred in 3 patients, 2.5, 3 and 13 months respectively after radiotherapy.

We initiated the current phase-II study to further evaluate toxicity and outcome of intensity-modulated whole-abdominal radiotherapy. The primary objective of the OVAR-IMRT-02 trial is to demonstrate the good tolerability this intensive multimodal adjuvant treatment in a cohort of 37 patients with advanced ovarian-cancer FIGO stage III, optimally debulked and with a complete remission after chemotherapy. Secondary objectives are rate of therapy stop, rate of therapy interruption, rate of acute toxicity, rate of late toxicity, rate of relapse-free survival, rate of overall-survival and quality of life (EORTC-QLQ30).

\section{Methods/design \\ Study design}

The OVAR-IMRT-02 is designed as a single-center one arm prospective phase II trial. In order to accelerate the 
data collection we plan to associate a second study center. Patients fulfilling the inclusion criteria will be treated with intensity-modulated WAR up to a total dose of 30 Gy in 1.5 Gy fractions within a consolidation concept. Thirty seven patients are planned to be enrolled.

\section{Study objectives}

The aim of the study is to demonstrate superior tolerability of consolidating intensity-modulated WAR compared to the conventional technique.

To get an impression of the tolerability of current radiation approaches, we explored the results from historical data on conventional radiation procedure. Adjuvant WAR using the conventional technique after radical surgery and chemotherapy showed a high rate of acute side effects (especially hematotoxicity, but also nausea, vomiting and diarrhoe). The pronounced acute toxicity, which is described in the literature as "severe", leads in many cases to a stop (10-15\%) or to an interruption (15-30\%) of the therapy and with it to a reduction of its efficacy $[22,23,32,33]$. This is one of the reasons why WAR is no longer applied. Within the OVAR-IMRT-01 Study, none of the 10 patients showed an acute toxicity CTC grade $4(0 \%)$, and the CTC grade 3 toxicity rate was $40 \%$.

Primary outcome variable of the OVAR-IMRT-02 Study is the tolerability of therapy, defined by the nonoccurrence of CTC grade 4 toxicity during the radiation therapy and until 6 weeks after its termination.

Secondary outcome variables are the rate of therapy stop, rate of therapy interruption, rate of acute toxicity, rate of late toxicity, rate of relapse-free survival, rate of overall survival and quality of life (EORTC-QLQ30).

\section{Trial organisation}

The trial OVAR-IMRT-02 is an investigator initiated trial. The trial is carried out by the Department of Radiation Oncology at the University of Heidelberg in co-operation with the Department of Gynaecology and Obstetrics. Statistical analysis and data management are performed by the Institute of Medical Biometry and Informatics at the University of Heidelberg. The trial is partly financially supported by the German Cancer Aid (Deutsche Krebshilfe).

\section{Coordination}

The overall coordination is performed by the Department of Radiation Oncology at the University of Heidelberg.

\section{Patient selection: Inclusion criteria}

Patients meeting all the following criteria will be considered for admission to the trial:
- histologically confirmed ovarian cancer or tube cancer or primary peritoneal carcinoma stage FIGO III

- status post primary optimal debulking surgery

- postoperative gross residual tumor $\leq 1 \mathrm{~cm}$ (R0, R1 or $\mathrm{R} 2<1 \mathrm{~cm}$ situation)

- status post (neo-) adjuvant chemotherapy with platin and taxane

- complete remission after adjuvant chemotherapy (clinically, radiographically and biochemically)

- Karnofsky performance score > 60

- patient $>18$ years of age

- written informed consent

\section{Patient selection: Exclusion criteria}

- stage FIGO I or II

- stage III with postoperative gross residual tumor > $1 \mathrm{~cm}$

- stage FIGO IV

- recurrence situation

- delayed wound healing post laparotomy

- leucopenia <2000/ml before radiotherapy

- thrombocytopenia $<75000 / \mathrm{ml}$ before radiotherapy

- clinically active renal, hepatic, cardiac, metabolic, respiratory, coagulation or hematopoietic disease

- status post pelvic or abdominal radiotherapy

- status post other cancer disease in the past 5 years (cervical cancer in situ, basal cell carcinoma, squamous cell carcinoma of the skin are excluded)

- participation in another clinical trial

\section{Statistical Design and Methods}

The primary endpoint of the trial is the tolerability rate, which is calculated as the percentage of patients for which no CTC Grade 4 toxicity occurs during the radiation therapy and until 6 weeks after its termination. The target tolerability rate is $90 \%$, and a tolerability rate of less or equal to $70 \%$ is considered to be not of interest. Denoting the tolerance rate by $p$, the related test problem can be formulated as $H_{0}: p \leq p_{0}=0.70$ versus $H_{1}$ : $p \geq p_{1}=0.90$ which will be assessed at one-side significance level $\alpha=0.05$.

The trial is designed as a two-stage study with a planned interim analysis, allowing for early stopping, and a power of 0.90 to reject the null hypothesis. In the first stage, 15 patients are enrolled. If all these patients would tolerate radiation therapy according to the definition of the primary endpoint, the study would stop with rejection of the null hypothesis. The study would terminate with acceptance of the null hypothesis, if less than 12 of the first 15 patients would tolerate the radiation 
therapy. According to the current design, the trial would continue with the second stage enrolling further 22 patients, if the number of patients that tolerate radiation therapy is between 12 and 14. In the final analysis, the null hypothesis can be rejected if the product of the one-sided p-values of the two stages is lower or equal to 0.021 . With an expected sample size of 21.4 for a tolerability rate of $p_{0}=0.70$ (i.e., under the null hypothesis), this design shows similar characteristics as Simon's (1989) optimal design whose expected sample size is 21.2. under the same scenario [34]. However, the adaptive two-stage design implemented in this trial allows a data-driven modification of the sample size of the second stage based on the results of the interim analysis or on external information while the specified significance level is controlled when the decision rules defined above are adhered to [35].

Additionally to the hypothesis testing approach, the primary endpoint will be analysed by applying Bayesian methods. Starting with the uniform distribution for the tolerability rate as prior distribution for the preceding trial OVAR-IMRT-01 [26,30,31], the posterior distribution of this trial is derived which serves as prior distribution for the current study OVAR-IMRT-02. Employing the results of OVAR-IMRT-02 leads to an updated posterior distribution for the tolerability rate from which Bayesian inference on $p$ will be derived.

The secondary outcome variables will be evaluated by application of methods of descriptive data analysis. Appropriate measures of the empirical distribution as well as $95 \%$ confidence intervals will be calculated. All statistical analyses will be performed with SAS version 9.0 or higher.

\section{Investigation schedule \\ Therapy Indication}

For each patient, the oncologic treatment concept after diagnosis is based on a tumor board review, following approved standard therapies and current guidelines. Consent of the colleagues from gynaecology, internal medicine and radiation oncology are obligatory.

\section{Pre-therapeutic examinations}

A complete staging is performed before surgery and/or chemotherapy, including abdominopelvic CT scan or MRI, radiography or CT scan of the chest, liver sonography, pelvic examination, transvaginal sonography, CA-125. After completion of chemotherapy, the complete remission is assessed by pelvic examination, transvaginal sonography and measure of CA-125.

The baseline visit is performed at study enrollment, after obtaining of the written consent of the patient. It includes blood test, clinical examination and quality of life questionnaire (EORTC-QLQ30).

\section{Radiotherapy-planning}

After immobilisation in a vacuum mattress and a scotch cast head mask (arms above the head) the patients undergo a native and contrast enhanced abdominopelvic computed tomography (optionally with 4D respiratory triggering). This CT scan is used for the restaging and for the radiation planning.

The clinical target volume (CTV) includes the whole peritoneal cavity extending from diaphragm to douglas cavity, the liver surface, and the pelvic and para-aortic node regions. The planning target volume (PTV) encompasses an axial margin of $1.5 \mathrm{~cm}$ around the CTV $(2.5 \mathrm{~cm}$ in the cranial direction). Organs at risk are kidneys, liver (except the $1 \mathrm{~cm}$-outer border), lungs, bones (vertebral bodies and pelvic bones), hear and spinal cord.

A dose of $30.0 \mathrm{~Gy}$ is prescribed to the median of the PTV. Inverse radiation planning is performed according to the national DIN norm and international recommendations (ICRU 50 Report 1999). The goal of optimization is to deliver a dose distribution in the PTV as homogeneous as possible in spite of maximal sparing of OARs with a high priority on liver, kidney and bone marrow. Tolerated maximum doses to OARs are not to exceed the TD5/5 for each organ (31).

\section{Radiation therapy}

Irradiation is applied as helical intensity-modulated radiation therapy (IMRT) using a tomotherapy device (Madison, Wisconsin, USA) with $6 \mathrm{MeV}$ photons. Control of positioning accuracy is performed daily with the integrated megavoltage computed tomography for tomotherapy (MV-CT scan, 3.5 MV).

\section{Duration of treatment}

The delay between the last chemotherapy and the onset of radiation should not exceed 10 weeks. The duration of the treatment planning is estimated about 2 or 3 weeks. The duration of radiotherapy is 4 weeks (20 fractions). The overall daily treatment time is expected to be about 15 to 20 minutes with a mean daily "time on table" of about half an hour.

\section{Treatment schedule}

The radiotherapy is performed on an out-patient basis with the possibility of hospital admission if required.

\section{Monitoring during treatment}

Patients are evaluated weekly during radiotherapy. Antiemetic and antidiarrheal medications are prescribed as required. Blood count is assessed twice weekly. Toxicities are graded according to the National Cancer Institute Common Toxicity Criteria version 3.0. The quality of life is assessed at the last treatment fraction using EORTC-QLQ30 questionnaire. The documentation of acute hematologic, genito-urinary and gastro-intestinal acute toxicities is especially relevant. 


\section{Follow up}

Follow-up visits are scheduled in addition to the standard gynaecology follow-up program at 6 weeks, 3, 6, 9, $12,15,18,24,30$ and 36 months, respectively, after completion of radiotherapy. Each visit includes blood test, CA-125, pelvic examination, transvaginal ultrasound, recording of adverse events and assessment of quality of life (questionnaire EORTC-QLQ30). The documentation of late genito-urinary and gastro-intestinal toxicities (such as bowel obstruction) is especially relevant. Additionally, a contrast-enhanced abdominopelvic CT scan or MRI will be performed at 6, 12 und 24 months, respectively.

\section{Duration of the study}

The primary objective of the study is to prove the good treatment tolerance, defined as non-occurrence of CTC grade 4 toxicities during radiotherapy and till 6 weeks after its completion. To assess the primary endpoint, the final study visit will be 6 weeks after the last patient completed the radiotherapy. The study ends three years after the last patient was treated. Recruitment of the patients is planned over a time period of 36 months; follow-up duration will be 36 months.

\section{Data Handling, Storage and Archiving of Date}

All findings including clinical and laboratory data will be documented by the investigator or an authorized member of the study team in the subject's medical record and in the CRF. The data will be stored and archived according to the $\$ 13$ of the German GCP-Regulation and $\$ 28$ c of the German X-Ray Regulation (StrlSchV) for at least 30 years after the trial termination.

\section{Ethics, informed consent and safety}

The final protocol was approved by the ethics committee of the University of Heidelberg, Heidelberg, Germany (Nr: S-353/2008). Additionally, a positive vote of the Bundesamt für Strahlenschutz (BfS) has been obtained. This study complies with the Helsinki Declaration in its recent German version. The trial will also be carried out in keeping with local legal and regulatory requirements. The ClinicalTrials.gov Protocol ID is NCT01180504.

All severe adverse events have to be reported immediately to the principal investigator. In addition, side effects and patient safety will be monitored by an independent data safety monitoring committee (DMC), composed of one radio oncologist, one gynaecologist and one statistician. In case of frequent or particularly serious adverse events, then the DMC would have to consider termination of the study. This evaluation has to be made in consideration of risk/benefit. The DMC will meet on a regular basis.

\section{Discussion}

The prognosis for patients with advanced FIGO stage III epithelial ovarian cancer remains poor despite the aggressive standard treatment, consisting of maximal cytoreductive chemotherapy and platinum-based chemotherapy. The median time to recurrence, which occurs mostly intraperitoneally, is less than 2 years, with a 5 -years survival rate of $-20-25 \%$ [1-5].

Ovarian cancer is a radiosensitive tumor, so that WAR as a consolidation therapy would appear to be a logical strategy. WAR used to be the standard treatment after surgery before the chemotherapy era. However, WAR has been almost totally excluded from the treatment of ovarian cancer during the past decade because of its high toxicity $[9,10]$. Since toxicity can be managed by modern radiotherapy technique (IMRT), WAR provides a new promising option in the consolidation treatment of ovarian carcinoma in patients with complete pathologic remission after adjuvant chemotherapy [26,30].

In our previous phase-I study, we showed for the first time the clinical feasibility of intensity-modulated WAR in combination with modern chemotherapy and surgery [31]. It rendered an excellent coverage of the entire peritoneal cavity including frequent sites of abdominal recurrence like the liver capsule and the diaphragm as well as of the pelvic- and para-aortic node regions. An effective sparing of liver, kidneys and bone marrow allowed delivering the treatment as planned, with a high compliance of patients.

The current phase-II succeeds to the phase-I study to demonstrate the good tolerability of the treatment (defined as non-occurrence of CTC grade 4 acute toxicities) and to further evaluate toxicity and outcome of this new treatment.

In the previous phase-I study, small bowel obstruction due to adhesions occurred in 3 of 10 patients, 2.5, 3 and 13 months respectively after radiotherapy. The exact role played by radiotherapy in the development of peritoneal adhesions remains unclear and further studies are needed to evaluate this problem. Risk factors for late bowel toxicity are suggested by literature: dose escalation with a total abdominal dose over $30 \mathrm{~Gy}$, pelvic-boost and/or para-aortic boost and previous second-look laparotomy are associated with a significant increase in small bowel obstruction [23,32,33]. Given that the addition of pelvic boost treatment is not clearly beneficial in the literature; the routine use of a pelvic boost in consolidation WAR should be questioned [23]. With respect to these arguments, this study was designed with careful attention to keeping the risk for late bowel toxicity as low as possible and bowel adverse events will be monitored accurately.

Intensity-modulated WAR could offer a new therapeutic option for consolidation treatment of advanced ovarian carcinoma after adjuvant chemotherapy in selected 
subgroups of patients. However, further consequent studies are needed to draw firm conclusions regarding the value of consolidation radiotherapy in the multimodal treatment of ovarian carcinoma, including immunologic and biologic therapies in the future.

\section{Abbreviations}

CRF: Case Report Form; CTCAE: Common Terminology Criteria for Adverse Events; CTV: Clinical Target Volume; DMC: Data Monitoring Committee; FIGO: International Federation of Gynaecology and Obstetrics; GCP: Good Clinical Practice; IMRT: Intensity Modulated Radiation Therapy; PTV: Planning Target Volume; TD5/5: Tolerance Dose 5\% over 5 years; WAR: Whole-Abdominal Radiotherapy.

\section{Author details}

'Department of Radiation Oncology, University of Heidelberg, Im Neuenheimer Feld 400, 69120 Heidelberg, Germany. ${ }^{2}$ Institute of Medical Biometry and Informatics, University of Heidelberg, Im Neuenheimer Feld 305, 69120 Heidelberg, Germany. ${ }^{3}$ Department of Gynaecology and Obstetrics, University of Heidelberg, Voss Strasse 9, 69115 Heidelberg, Germany. ${ }^{4}$ National Center for Tumor Diseases, University of Heidelberg, Im Neuenheimer Feld 460, 69120 Heidelberg, Germany.

\section{Authors' contributions}

$\mathrm{NR}, \mathrm{MK}, \mathrm{AS}, \mathrm{WH}$ and JD planned, organized and conduct the study. NR, KL, $M E, A S$, and CS recruit the patients for the study. NR, FS and SK perform planning and radiation therapy. Medical care and follow up is provided by $N R$, FS, SK, KL, ME, AS and CS. Statistical analysis is carried out by MK. All authors have read and approved the final manuscript.

\section{Competing interests}

The authors declare that they have no competing interests.

Received: 18 November 2010 Accepted: 28 January 2011 Published: 28 January 2011

\section{References}

1. Bristow RE, Tomacruz RS, Armstrong DK, Trimble EL, Montz FJ: Survival effect of maximal cytoreductive surgery for advanced ovarian carcinoma during the platinum era: a meta-analysis. J Clin Oncol 2002, 20:1248-1259.

2. Cannistra SA, Bast RC Jr, Berek JS, Bookman MA, Crum CP, DePriest PD, et al: Progress in the management of gynecologic cancer: consensus summary statement. J Clin Oncol 2003, 21:129s-132s,

3. Lambert HE, Gregory WM, Nelstrop AE, Rustin GJ: Long-term survival in 463 women treated with platinum analogs for advanced epithelial carcinoma of the ovary: life expectancy compared to women of an agematched normal population. Int I Gynecol Cancer 2004, 14:772-778.

4. McGuire WP III: Current status of taxane and platinum-based chemotherapy in ovarian cancer. J Clin Oncol 2003, 21:133s-135s.

5. Ozols RF, Bundy BN, Greer BE, Fowler JM, Clarke-Pearson D, Burger RA, et al Phase III trial of carboplatin and paclitaxel compared with cisplatin and paclitaxel in patients with optimally resected stage III ovarian cancer: a Gynecologic Oncology Group study. J Clin Oncol 2003, 21:3194-3200.

6. Donato ML, Aleman A, Champlin RE, Saliba RM, Wharton JT, Burke TW, et al: Analysis of 96 patients with advanced ovarian carcinoma treated with high-dose chemotherapy and autologous stem cell transplantation. Bone Marrow Transplant 2004, 33:1219-1224.

7. Dufour P, Bergerat JP, Barats JC, Giron C, Duclos B, Dellenbach P, et al: Intraperitoneal mitoxantrone as consolidation treatment for patients with ovarian carcinoma in pathologic complete remission. Cancer 1994, 73:1865-1869.

8. Varia MA, Stehman FB, Bundy BN, Benda JA, Clarke-Pearson DL, Alvarez RD, et al: Intraperitoneal radioactive phosphorus (32P) versus observation after negative second-look laparotomy for stage III ovarian carcinoma: a randomized trial of the Gynecologic Oncology Group. I Clin Oncol 2003, 21:2849-2855

9. Dembo AJ: Abdominopelvic radiotherapy in ovarian cancer. A 10-year experience. Cancer 1985, 55:2285-2290.
10. Dembo AJ: Epithelial ovarian cancer: the role of radiotherapy. Int I Radiat Oncol Biol Phys 1992, 22:835-845.

11. Smith JP, Rutledge FN, Delclos L: Postoperative treatment of early cancer of the ovary: a random trial between postoperative irradiation and chemotherapy. Natl Cancer Inst Monogr 1975, 42:149-153.

12. Kapp KS, Kapp DS, Poschauko J, Stucklschweiger GF, Hackl A, Pickel H, et al: The prognostic significance of peritoneal seeding and size of postsurgical residual in patients with stage III epithelial ovarian cancer treated with surgery, chemotherapy, and high-dose radiotherapy. Gynecol Oncol 1999, 74:400-407.

13. Lambert HE, Rustin GJ, Gregory WM, Nelstrop AE: A randomized trial of five versus eight courses of cisplatin or carboplatin in advanced epithelial ovarian carcinoma. A North Thames Ovary Group Study. Ann Oncol 1997, 8:327-333.

14. MacGibbon A, Bucci J, MacLeod C, Solomon J, Dalrymple C, Firth I, et al: Whole abdominal radiotherapy following second-look laparotomy for ovarian carcinoma. Gynecol Oncol 1999, 75:62-67.

15. Pickel H, Lahousen M, Petru E, Stettner H, Hackl A, Kapp K, et al: Consolidation radiotherapy after carboplatin-based chemotherapy in radically operated advanced ovarian cancer. Gynecol Oncol 1999, 72:215-219.

16. Thomas GM: Is there a role for consolidation or salvage radiotherapy after chemotherapy in advanced epithelial ovarian cancer? Gynecol Oncol 1993, 51:97-103.

17. Thomas GM, Dembo AJ: Integrating radiation therapy into the management of ovarian cancer. Cancer 1993, 71:1710-1718.

18. Sorbe B: Consolidation treatment of advanced (FIGO stage III) ovarian carcinoma in complete surgical remission after induction chemotherapy: a randomized, controlled, clinical trial comparing whole abdominal radiotherapy, chemotherapy, and no further treatment. Int I Gynecol Cancer 2003, 13:278-286

19. Einhorn N, Lundell M, Nilsson B, Ragnarsson-Olding B, Sjovall K: Is there place for radiotherapy in the treatment of advanced ovarian cancer? Radiother Oncol 1999, 53:213-218.

20. Einhorn N, Trope C, Ridderheim M, Boman K, Sorbe B, Cavallin-Stahl E: A systematic overview of radiation therapy effects in ovarian cancer. Acta Oncol 2003, 42:562-566.

21. Fyles AW, Thomas GM, Pintilie M, Ackerman I, Levin W: A randomized study of two doses of abdominopelvic radiation therapy for patients with optimally debulked Stage I, II, and III ovarian cancer. Int J Radiat Oncol Biol Phys 1998, 41:543-549.

22. Dinniwell $R$, Lock $M$, Pintilie $M$, Fyles $A$, Laframboise $S$, Depetrillo $D$, et al: Consolidative abdominopelvic radiotherapy after surgery and carboplatin/paclitaxel chemotherapy for epithelial ovarian cancer. Int J Radiat Oncol Biol Phys 2005, 62:104-110.

23. Whelan TJ, Dembo AJ, Bush RS, Sturgeon JF, Fine S, Pringle JF, et al: Complications of whole abdominal and pelvic radiotherapy following chemotherapy for advanced ovarian cancer. Int I Radiat Oncol Biol Phys 1992, 22:853-858

24. Fuller DB, Sause WT, Plenk HP, Menlove RL: Analysis of postoperative radiation therapy in stage I through III epithelial ovarian carcinoma. J Clin Oncol 1987, 5:897-905.

25. Duthoy W, De Gersem W, Vergote K, Coghe M, Boterberg T, De Deene Y, et al: Whole abdominopelvic radiotherapy (WAPRT) using intensitymodulated arc therapy (IMAT): first clinical experience. Int I Radiat Oncol Biol Phys 2003, 57:1019-1032.

26. Rochet N, Sterzing F, Jensen A, Dinkel J, Herfarth K, Schubert K, et al: Helical tomotherapy as a new treatment technique for whole abdominal irradiation. Strahlenther Onkol 2008, 184:145-149.

27. Debus J, Zierhut D, Didinger B, Schlegel W, Wannenmacher M: Inverse planning and intensity-modulated radiotherapy in patients with prostate cancer. Front Radiat Ther Oncol 2002, 36:25-34.

28. Hong L, Alektiar K, Chui C, LoSasso T, Hunt M, Spirou S, et al: IMRT of large fields: whole-abdomen irradiation. Int J Radiat Oncol Biol Phys 2002, 54:278-289.

29. Mackie TR, Holmes T, Swerdloff S, Reckwerdt P, Deasy JO, Yang J, et al: Tomotherapy: a new concept for the delivery of dynamic conformal radiotherapy. Med Phys 1993, 20:1709-1719.

30. Rochet $N$, Jensen AD, Sterzing F, Munter MW, Eichbaum MH, Schneeweiss $A$, et al: Adjuvant whole abdominal intensity modulated radiotherapy (IMRT) for high risk stage FIGO III patients with ovarian 
cancer (OVAR-IMRT-01) - Pilot trial of a phase I/II study: study protocol. BMC Cancer 2007, 7:227.

31. Rochet N, Sterzing F, Jensen AD, Dinkel J, Herfarth KK, Schubert K, et al: Intensity-modulated whole abdominal radiotherapy after surgery and carboplatin/taxane chemotherapy for advanced ovarian cancer: phase study. Int J Radiat Oncol Biol Phys 2010, 76:1382-1389.

32. Firat S, Murray K, Erickson B: High-dose whole abdominal and pelvic irradiation for treatment of ovarian carcinoma: long-term toxicity and outcomes. Int J Radiat Oncol Biol Phys 2003, 57:201-207.

33. Fyles AW, Dembo AJ, Bush RS, Levin W, Manchul LA, Pringle JF, et al: Analysis of complications in patients treated with abdomino-pelvic radiation therapy for ovarian carcinoma. Int J Radiat Oncol Biol Phys 1992, 22:847-851.

34. Simon R: Optimal two-stage designs for phase II clinical trials. Control Clin Trials 1989, 10:1-10.

35. Englert S, Kieser M: Adaptive designs for single-arm, phase II trials in oncology, Internal Report. 2010.

Pre-publication history

The pre-publication history for this paper can be accessed here:

http://www.biomedcentral.com/1471-2407/11/41/prepub

doi:10.1186/1471-2407-11-41

Cite this article as: Rochet et al:: Phase II study evaluating consolidation whole abdominal intensity-modulated radiotherapy (IMRT) in patients with advanced ovarian cancer stage FIGO III - The OVAR-IMRT-02 Study. BMC Cancer 2011 11:41.

\section{Submit your next manuscript to BioMed Central and take full advantage of:}

- Convenient online submission

- Thorough peer review

- No space constraints or color figure charges

- Immediate publication on acceptance

- Inclusion in PubMed, CAS, Scopus and Google Scholar

- Research which is freely available for redistribution

Submit your manuscript at www.biomedcentral.com/submit 